\title{
РАЗРАБОТКИ ИСТОЧНИКОВ ТОКА С РАДИОАКТИВНЫМИ ИЗОТОПАМИ НА ОСНОВЕ СУПЕРКОНДЕНСАТОРОВ (ИТРИ): ТЕОРИЯ, ЭКСПЕРИМЕНТ, ПЕРСПЕКТИВЫ
}

\author{
В. А. Степанов, В.А. Чернов, Ю. Г. Паршиков, В. П. Лебедев, Е. В. Харанжевский \\ ВЛАДИМИР АЛЕКСАНДРОВИЧ СТЕПАНОВ - д.ф.-м.н., профессор Обнинского института атомной \\ энергетики Научно-исследовательского ядерного университета МИФИ, ведущий научный сотрудник \\ ФГБУН Межведомственного иентра аналитических исследований в области физики, химии и биологии \\ при Президиуме PAH. E-mail: stepanov@iate.obninsk.ru.
}

ВЛАДИМИР АЛЕКСЕЕВИЧ ЧЕРНОВ - к.ф.-м.н., доиент, заместитель начальника лаборатории по науке ФГУП ГНЦ РФ Физико-энергетического института им. А.И. Лейпунского, старший научный сотрудник ФГБУН Межведомственного иентра аналитических исследований в области физики, химии и биологии при Президиуме PAH.E-mail: vac.lnfi@ippe.ru.

ЮРИЙ ГРИГОРЬЕВИЧ ПАРШИКОВ - д.т.н., директор ФГБУН Межведомственного иентра аналитических исследований в области физики, химии и биологии при Президиуме PAH. E-mail: mzairan@mzairan.ru.

ВИКТОР ПЕТРОВИЧ ЛЕБЕДЕВ - к.т.н., советник генерального директора ОАО «ЭЛЕКОНД».

E-mail:lebedev18@live.ru.

ЕВГЕНИЙ ВИКТОРОВИЧ ХАРАНЖЕВСКИЙ - д.т.н., профессор Удмуртского государственного университета, главный конструктор по накопителям энергии ОАО «ЭЛЕКОНД».E-mail: eh@udsu.ru.

249040 Калужская обл., г. Обнинск, Студгородок, д. 1. Обнинский институт атомной энергетики НИЯУ МИФИ.

117997 г. Москва, ул. Профсоюзная, д. 65, стр. 6. ФГБУН Межведомственньй центр аналитических исследований в области физики, химии и биологии при Президиуме РАН.

249033 Калужская обл., г. Обнинск, пл. Бондаренко, д. 1. ФГУП «ГНЦ РФ-ФЭИ» им. А.И. Лейпунского.

427968 Удмуртская Республика, г. Сарапул, ул. Калинина, д.3. ОАО «ЭЛЕКОНД».

426034 г. Ижевск, ул. Университетская, 1. Удмуртский государственный университет.

Исследованы суперконденсаторы с активированными нейтронами введенных в электроды веществ. Показано, что суперконденсаторы с внутренней радиоактивностью могут быть эффективными источниками тока с радиоактивныли изотопами ИТРИ. Наиболее вероятный механизм разделения и накопления электрического заряда в ИТРИ связан с неравновесными термоэлектрическими явлениями в приэлектродных нанообластях.

Ключевые слова: источник тока с радиоактивным изотопом, суперконденсатор, нейтронное облучение, радиационно-индуцированный электрический заряд. 


\title{
DEVELOPMENT OF CURRENT SOURCES WITH RADIOACTIVE ISOTOPES BASED ON SUPERCAPACITORS (CSRI): THEORY, EXPERIMENT, PROMISE
}

\author{
V.A. Stepanov ${ }^{1,2}$, V.A. Chernov ${ }^{2,3}$, Yu. G. Parshikov' , V.P. Lebedev ${ }^{4}$, E. V. Kharanzhevskiy ${ }^{4,5}$ \\ ${ }^{1} 1$ Studgorodok st., Kaluga rg., Obninsk, 249040, Russia. National research nuclear university (NRNU) MEPh. \\ ${ }^{2} 65$ Profsoyuznaya Street, Moscow, 117997, Russia. Interdepartment Center of Analytical Research in Physics, \\ Chemistry, and Biology, Presidium of Russian Academy of Sciences. \\ ${ }^{3} 1$ Bondarenko sq., Kaluga rg., Obninsk., 249033, Russia. Leypunsky Institute for Physics and Power Engineering. \\ 43, Kalinin Str., Sarapul, Udmurt Republic, 427968, Russia. Open Joint Stock Company “ELECOND” \\ ${ }^{5}$ 1, Universitetskaya St., bld. 1, Izhevsk, 426034, Russia. Udmurt State University.
}

\begin{abstract}
Supercapacitors with activated neutrons of substances in electrodes are investigated. It is shown that supercapacitors with internal radioactivity can be effective current sources with radioactive isotopes (CSRI). The most probable mechanism of separation and accumulation of electric charge in ITRI is associated with nonequilibrium thermoelectric phenomena in near-electrode nano-areas.
\end{abstract}

Key words: current source with radioactive isotope, supercapacitor, neutron irradiation, radiation-induced electric charge.

\section{Введение}

За последние полвека изобретено несколько типов прямых преобразователей ядерной энергии в электрическую (см., например, [1]). Одними из первых источников электроэнергии, в которых происходит преобразование энергии ядерных превращений, были автономные радиоизотопные термоэлектрические генераторы, содержащие соединения радиоактивных изотопов (Ро-210, $\mathrm{Pu}-238, \mathrm{Sr}-90$ и др.). В них энергия радиоактивного распада переходит в тепло, а затем тепловая энергия преобразуется в электрическую. Для преобразования тепловой энергии в таких генераторах используют термоэлектрические, термоэмиссионные или динамические устройства. В термоэлектрических устройствах используется эффект Зеебека возникновения ЭДС в термопарах при наличии градиента температуры. В термоэмиссионных устройствах разделение электрического заряда происходит в результате эмиссии электронов из нагретого катода. В динамических устройствах последовательность преобразования энергии дополняется механической стадией, на которой нагреваемое рабочее тело совершает механическую работу, кото- рая преобразуется в электрическую энергию. Радиоизотопные термоэлектрические генераторы имеют КПД 3-5 \%, мощность до 100 Вт и используются в космических аппаратах, в маяках, бакенах и медицине. Однако радиоизотопные термоэлектрические генераторы обладают удельной мощностью до 3 Вт/кг и весьма дороги в изготовлении.

В бета-вольтаических источниках питания содержатся бета-активные изотопы (Ni-63, Ce-144, Cs-137, Pm-147, Kr-85, Н-3 и др.). Испускаемые изотопами электроны или позитроны попадают на полупроводник, и в области p-n перехода происходит генерация электрон-дырочных пар, которые разделяются областью пространственного заряда. В результате на р- и n- поверхностях полупроводника возникает разность электрического потенциала. Период полураспада используемых изотопов может быть от 2,64 (Pm-147) до 100 (Ni-63) лет, поэтому срок службы бета-вольтаических источников может составлять годы и десятки лет. Удельная мощность бета-вольтаических источников питания может достигать 1 кВт/кг.

Активное использование указанных источников электроэнергии сдерживается чрезвычайной дороговизной реализуемых к настоящему времени 
различных конфигураций преобразователей энергии радиоактивных изотопов в электричество. Высокая цена обусловлена дороговизной выделения нужных изотопов ( $\beta$ или $\alpha$ активных) и сложностью работы с радиоактивным материалом. Изготовление одной функциональной батарейки на, например, ${ }^{63} \mathrm{Ni}$ ( $\beta$ - излучатель) обойдется в миллионы рублей. Даже перевод в серийное производство не приблизит цену известных автономных источников электроэнергии на разработанных принципах преобразования к цене химических источников. По этой причине источники энергии на радиоактивных изотопах использовали и используют, в основном, там, где большие затраты приемлемы (на космических объектах, в военной технике).

Суперконденсаторы с внутренней радиоактивностью могут быть эффективными ИТРИ источниками тока с радиоактивными изотопами в том случае, если имеется асимметрия электродов, либо геометрическая, например, по площади, либо по распределению/составу радиоактивных изотопов на электродах. Необходимый эффект от преобразования ядерной энергии в электрическую достигается чрезвычайно большой межфазной площадью, на которой происходит радиационно-индуцированное разделение заряда. Дорогостоящее изготовление систем с радиоактивными веществами заменяется ядерным легированием в нейтронном поле изготовленного в обычных заводских условиях полуфабриката, когда в результате взаимодействия с нейтронами введенные в материал электрода элементы превращаются в радиоактивные изотопы, и суперконденсатор превращается в ИТРИ.

\section{ИТРИ с наведенной радиоактивностью}

Физическое моделирование процессов разделения электрического заряда в ИТРИ было проведено с помощью измерений электрофизических характеристик суперконденсаторов, в которых роль радиоактивного изотопа играет проникающее непрерывное нейтронное излучение [2]. Облучали асимметричные по площади электродов суперконденсаторы с твердым неорганическим электролитом $\mathrm{Ag}_{4} \mathrm{RbJ}_{5}$, а в работах [3-5] с органическим электролитом (раствор TEATFB в пропиленкарбонате) Разделение и накопление электрического заряда на электродах наблюдали непосредственно в процессе нейтронного облучения. Было показано, что накопление заряда зависит от асимметрии электродов, не зависит от плотности потока нейтронов и определяется поглощенной дозой излучения. Характеристики ИТРИ, можно оценивать с помощью экспериментально полученной в $[2,6]$ величины удельной на единицу мощности поглощенной дозы поверхностной плотности электрического заряда, создаваемой на проводнике структуры металл-диэлектрик-метал (МДМ), $\rho \sim 10^{-8}$ Кл/Гр $\cdot \mathrm{m}^{2}$. Эта величина постоянна вне зависимости от типа МДМ-структуры и вида облучения (электроного, нейтронного, ионного).

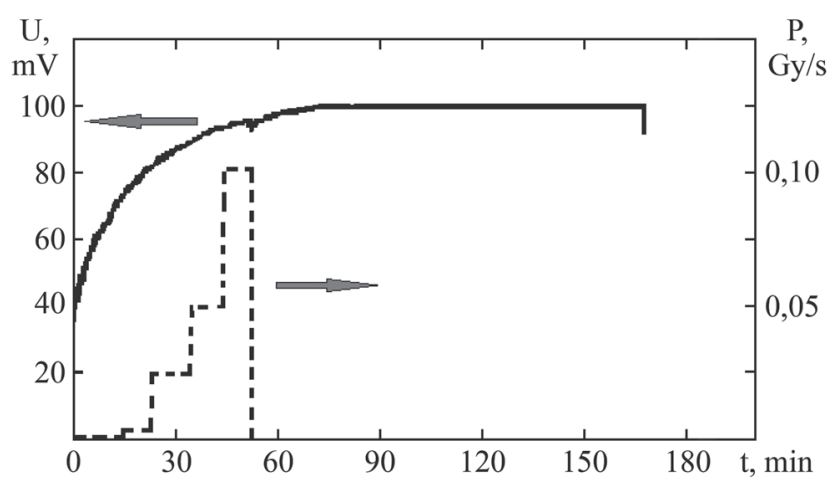

Рис. 1. Зависимости мощности поглощенной дозы от источника нейтронов Р и электрического напряжения между электродами $U$ асимметричного суперконденсатора с органическим электролитом

Оказалось, что электрическое напряжение между электродами и емкость асимметричных суперконденсаторах, в которых один из электродов представлял собой гладкую алюминиевую фольгу, увеличивались также и после прекращения облучения (рис. 1). Это связано с наведенной радиоактивностью, возникающей вследствие активации нейтронами неорганического и органического электролитов, а также углеродного материала электродов. В твердых электролитах возникают активные изотопы ${ }^{86} \mathrm{Rb},{ }^{85} \mathrm{Kr}$ [7]. $\beta$-активный изотоп ${ }^{14} \mathrm{C}$ возникает в органических электролитах и материале электродов.

Макеты ИТРИ с $\beta$-активностью одного из электродов, которая возникала за счет нейтронной активации предварительно введенных веществ были изготовлены в ОАО «ЭЛЕКОНД». Электроды с эффективной площадью $2000 \mathrm{~m}^{2}$ состояли из активированного угля со связующими, а органический электролит представлял собой раствор ТЕАТFВ в пропиленкарбонате с различной молярностью. Один из электродов ИТРИ содержал наночастицы

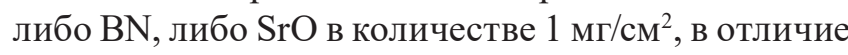
от суперконденсатора (СК), в котором оба электрода были одинаковы. После облучения нейтронами в результате реакций ${ }^{14} \mathrm{~N}(\mathrm{n}, \mathrm{p}){ }^{14} \mathrm{C}$ и ${ }^{88} \mathrm{Sr}(\mathrm{n}, \mathrm{g}){ }^{89} \mathrm{Sr}$ в материалах электродов появлялись $\beta$-активные изотопы углерода или стронция. Результаты измерений разности потенциалов на облученных образ- 


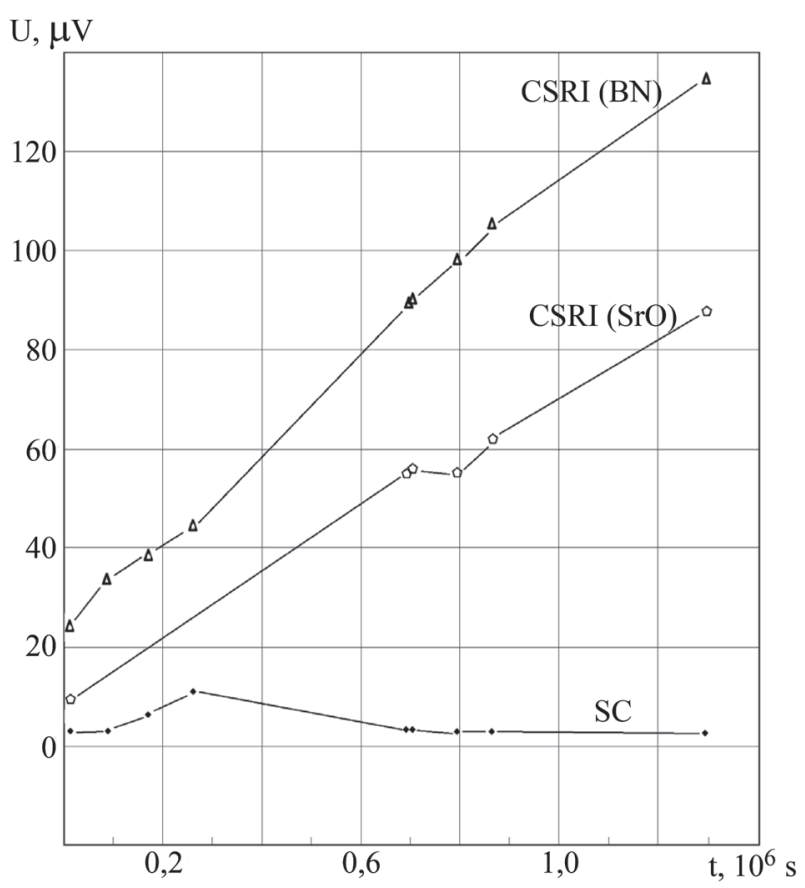

Рис. 2. Зависимости разности потенциалов от времени облученных нейтронами с дозой $2,9 \cdot 10^{13} \mathrm{H} / \mathrm{cm}^{2}$ образцов источников тока CSRI и суперконденсатора SC

цах СК и ИТРИ с $\mathrm{BN}$ и $\mathrm{SrO}$ представлены на рисунке 2 . Видно, что после облучения нейтронами дозой около $2,9 \cdot 10^{13} \mathrm{H} / \mathrm{cm}^{2}$, за время $1,3 \cdot 10^{6} \mathrm{c}$ в ИТРИ разность потенциалов возросла до 70-130 мкВ. В течение того же времени разность потенциалов на СК не изменяется с точностью 5 мкВ.

Для исследования влияния дозы облучения и содержания $\beta$-активных изотопов на скорость изменения разности потенциалов на электродах образец ИТРИ(BN) дополнительно облучили до дозы $1,0 \cdot 10^{15} \mathrm{H} / \mathrm{cm}^{2}$. Перед этим электроды макета были замкнуты на $10^{4}$ с. После облучения через 17 суток выдержки для спада наведенной короткоживущей гамма-активности от элементов конструкции электроды ИТРИ(BN) также замкнули на 4 суток $\left(3,5 \cdot 10^{5}\right.$ c). Результаты последующих в течение 21 суток $\left(1,8 \cdot 10^{6}\right.$ с) измерений разности потенциалов показаны на рисунке 3 . К началу измерений разность потенциалов составила $20 \mathrm{M \kappa B}$, а через $3 \cdot 10^{5}$ с медленный рост напряжения, как видно на рисунке, сменился быстрым. Интересно, что с уменьшением молярности органического электролита, от 1 до 0,01 М, уменьшается скорость заряжения ИТРИ, а на больших временах увеличение скорости зарядки сменяется ее падением вплоть до полной разрядки ИТРИ.

Важным экспериментальным подтверждением реализации ИТРИ на основе суперконденсато-

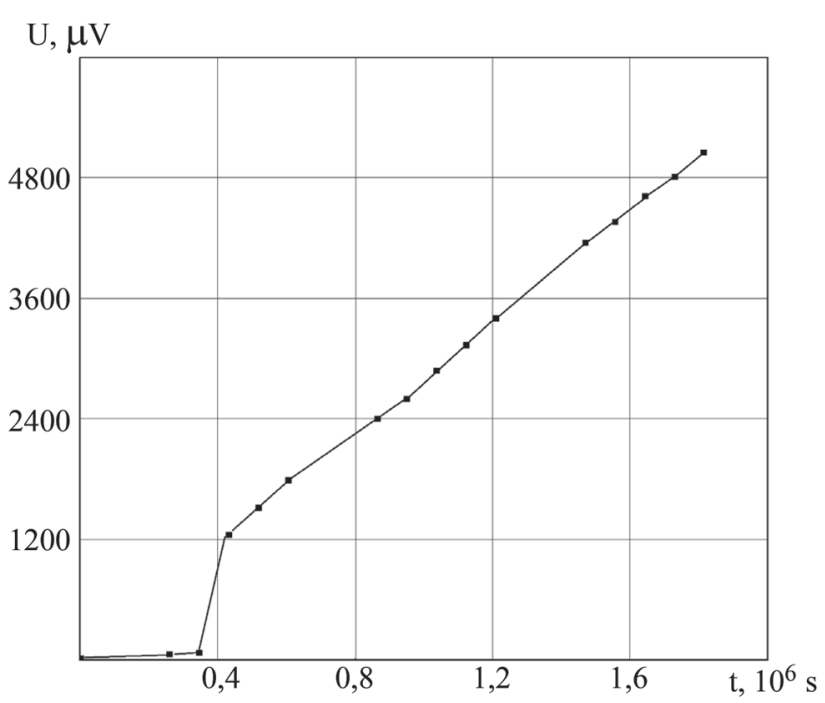

Рис. 3. Изменение разности потенциалов облученного нейтронами с дозой $1,0 \cdot 10^{15} \mathrm{H} / \mathrm{cm}^{2}$ образца CSRI(BN)

ра является результат изменения скорости зарядки с увеличением активационной нейтронной дозы. Сравнение графиков на рисунках 2 и 3 показывает, что скорость зарядки образца ИТРИ(ВN) увеличилась в 31 раз от 7,8 мкВ/сут до 240 мкВ/сут, при увеличении дозы облучения нейтронами в 34,5 раза. Это свидетельствует о пропорциональности скорости разделения заряда количеству $\beta$-активных изотопов ${ }^{14} \mathrm{C}$ в электроде суперконденсатора.

Некоторые выводы относительно микроскопического механизма разделения зарядов в макетах ИТРИ можно сделать, анализируя кинетику изменения разности потенциалов на электродах образцов. В течение $10^{6}$ с разность потенциалов увеличивается на 100 мкВ (рисунок 2), что соответствует скорости накопления заряда $\sim 10^{-8}$ Кл/с. С другой стороны, можно оценить число распадов в единицу времени получаемых активацией нейтронами изотопов. С учетом известных данных о сечении реакции ${ }^{14} \mathrm{~N}(\mathrm{n}, \mathrm{p}){ }^{14} \mathrm{C}$ [7] и энергии нейтронов в диапазоне энергий от тепловых до $10^{4}$ эВ получим, что при дозе $2,9 \cdot 10^{13} \mathrm{H} / \mathrm{cm}^{2}$ доля активных ядер ${ }^{14} \mathrm{C}$ составляет $\sim 10^{-11}$. Так как содержание $\mathrm{BN}$ составляет 40 мг, это соответствует $5 \cdot 10^{20}$ атомам азота на всем электроде и образовавшимся $5 \cdot 10^{9}$ изотопам углерода. Ядра ${ }^{14} \mathrm{C}$ испытывают $\beta$-распад с периодом 5700 лет $\left(1,8 \cdot 10^{11} \mathrm{c}\right)$, что означает $\sim 1$ распад за 100 с на всем электроде ИТРИ(BN). Если полагать, что все ядерные реакции приводят к разделению заряда между электродом и электролитом, то один распад приводит к перемещению через приграничный двойной электрический слой $\sim 10^{13}$ электронов. 
Аналогичный результат получается для ИТРИ( $\mathrm{SrO})$. Эффективное среднее сечение ${ }^{88} \mathrm{Sr}(\mathrm{n}, \mathrm{g}){ }^{89} \mathrm{Sr}$ [7] для спектра нейтронов составляет 3 барн, так что доля активированных изотопов также $\sim 10^{-11}$. С учетом общего числа атомов $\mathrm{Sr} 5 \cdot 10^{19}$ и периода полураспада ${ }^{89} \mathrm{Sr} 54$ дня $\left(4,7 \cdot 10^{6}\right.$ c) получим оценку в несколько десятков делений за секунду. Это соответствует перемещению до $10^{9}-10^{10}$ электронов через границу углеродный слой - электролит в результате одного ядерного превращения.

Такая оценка с учетом выделяющейся при $\beta$-распаде энергии 0,155 МэВ $\left({ }^{14} \mathrm{C}\right)$ и 1,5 МэВ $\left({ }^{89} \mathrm{Sr}\right)$ исключает механизмы разделения заряда в результате ионизации - рождения вторичных электронов, образования электронов и дырок и т.П. Действительно, число вторичных электронов при энергии ионизации более 1эВ не может превышать числа $10^{6}$. Кроме того, оценки для ИТРИ с электродами с $\mathrm{BN}$ и $\mathrm{SrO}$ отличаются на 3-4 порядка, в то время как из эксперимента следует, примерно, одинаковый результат. Вероятнее всего, механизм разделения и накопления электрического заряда в ИТРИ связан с микроскопическими неравновесными явлениями термоэлектронного переноса через двойной, толщиной в несколько межатомных расстояний, электрический слой на границе с электролитом.

Выделяющаяся при $\beta$-распаде энергия приводит к увеличению температуры электронов в нанообластях на поверхности проводящего углеграфитового электрода до 1-2 эВ. На временах до $10^{-13}$ с до таких же величин возрастает и решеточная температура, и возникает, так называемый, «термопик», который может существовать на временах до $10^{-6}-10^{-7}$ с в наноразмерных кристаллитах. Оценки в соответствии с [8] с использованием газодинамических соотношений переноса в условиях, возникающих на нанометровом масштабе «градиентов температуры» до $10^{14}$ К/м приводят к значениям скорости «испарения» электронов из нанокристаллов на уровне до $10^{13}$ на временнах $\sim 10^{-11} \mathrm{c}$.

Процесс является стохастическим и сильно неоднородным вдоль поверхности раздела, движущей силой которого являются локальные всплески «температуры» до 1-2 эВ. В течение таких «термопиков» через двойной электрический слой перемещается до $10^{13}$ электронов. Неоднородность распределения заряда вдоль поверхности (неоднородность образующегося двойного электрического слоя) приводит к длительным процессам перетекания, выравнивания заряда в приповерхностном слое электролита, что, вероятно, обуславливает начальное замедление процесса заряжения ИТРИ в течение $3 \cdot 10^{5} \mathrm{c}$, которое хорошо видно на рисунке 3. Длительная релаксация в процессах перезарядки суперконденсаторов - хорошо известный факт $[9,10]$.

\section{Динамическая неустойчивость в суперконденсаторах после нейтронного облучения}

Для понимания механизмов радиационно-индуцированного разделения заряда в ИТРИ, процессов формирования двойного электрического слоя в электролите на неоднородной поверхности углеродных электродов, устойчивости такого слоя в условиях внутренней радиоактивности, динамики разрядки и зарядки и т.д. необходимы длительные наблюдения за суперконденсаторами, в которых после облучения имеется внутренняя активность за счет наличия радиоактивных изотопов в материале электрода или в электролите. Такие наблюдения были проведены за асимметричными по площади электродов суперконденсаторами с емкостью 22 Ф с твердым неорганическим электролитом $\mathrm{Ag}_{4} \mathrm{RbJ}_{5}$ после импульсного реакторного облучения (доза 2 Гр по быстрым нейтронам). Асимметрия была связана с различием в $10^{3} \mathrm{M}^{2}$ площадей электродов на основе нанопористого углерода.

После импульсного реакторного облучения $\left(2 \cdot 10^{4} \Gamma \mathrm{p} / \mathrm{c}, 1 \cdot 10^{16} \mathrm{H}, \gamma / \mathrm{cm}^{2} \mathrm{c}\right)$ с поглощенной дозой 2 Гр изменение напряжение на суперконденсаторах составило от 26 до 135 мВ [2]. Было показано, что изменение удельной электрической энергии суперконденсаторов (до $10^{2}$ Дж/кг) значительно превышает поглощенную энергию от реакторного импульса (2 Гр), так что электрическое заряжение связано с нейтронной активацией материалов элементов суперконденсаторов.

Было проведено повторное облучение двух суперконденсаторов, в результате чего суммарная доза составила $2 \cdot 10^{12}$ н/см² (4 Гр). Наведенная нейтронами радиоактивность возникает вследствие активации нейтронами неорганического диэлектрика $\mathrm{Ag}_{4} \mathrm{RbJ}_{5}$ и углеродного материала электродов. В электролите в результате ${ }^{85} \mathrm{Rb}(\mathrm{n}, \gamma){ }^{86} \mathrm{Rb}$ реакции возникает активный изотоп с периодом полураспада 18,642 сут. ( $\beta$ 1,8 и 0,7 МэВ, $\gamma 1,08$ МэВ), a в результате ${ }^{85} \mathrm{Rb}(\mathrm{n}, \mathrm{p}){ }^{85} \mathrm{Kr}$ реакции получается изотоп с периодом распада $10,776(3)$ лет ( $\beta$ 0,72 МэВ и $\gamma$ 0,54 МэВ) [7]. $\beta$-активный (0,155 МэВ) изотоп ${ }^{14} \mathrm{C}$ с периодом распада 5700 лет возникает в материале электродов в результате $(\mathrm{n}, \gamma)$ реакции из ${ }^{13} \mathrm{C}$, присутствующего в количестве 1,07\% в природном углероде, а также из растворенного азота в результате реакции ${ }^{14} \mathrm{~N}(\mathrm{n}, \mathrm{p}){ }^{14} \mathrm{C} . \gamma$-активность 


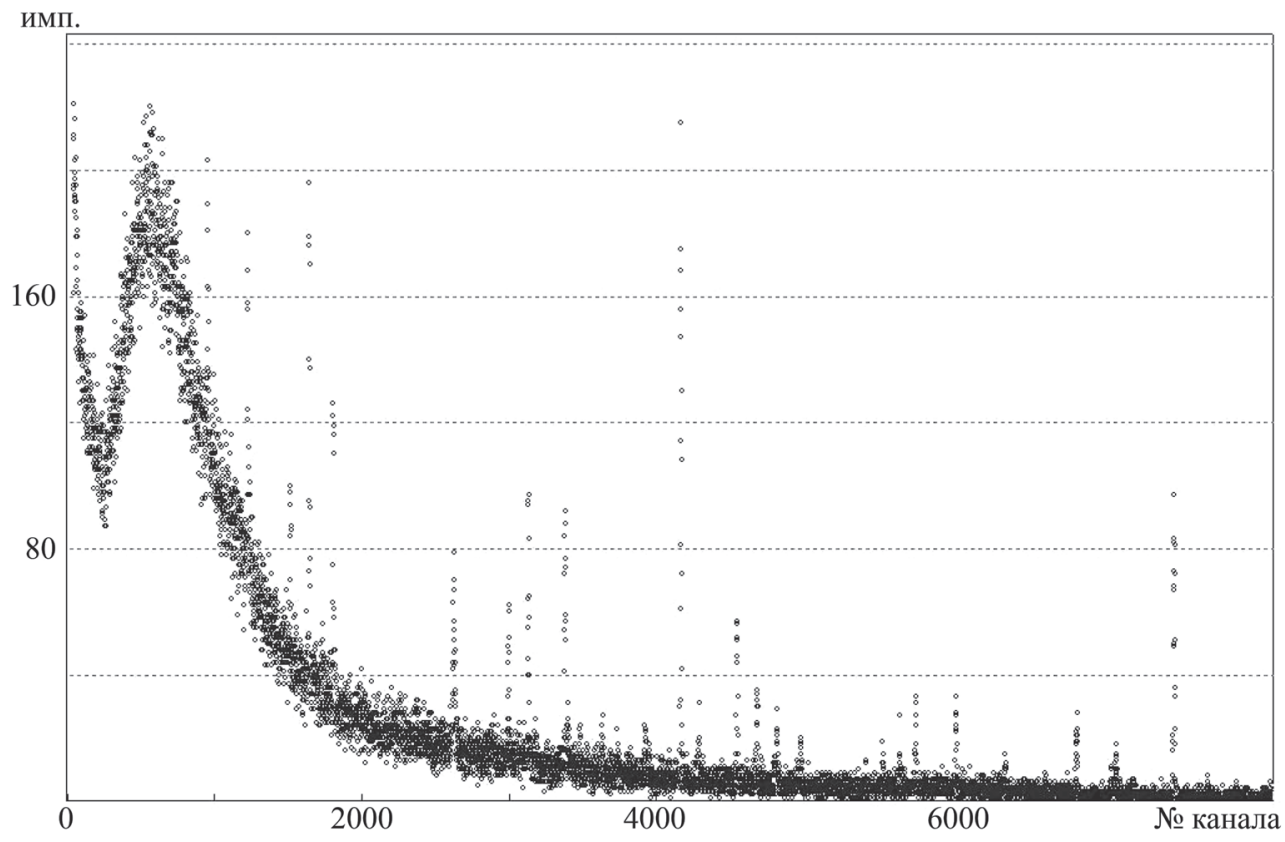

Рис. 4. Спектр гамма-излучения, облученного ионистора

возникающих изотопов обнаруживается в результате спектрометрических измерений. На рис. 4 приведен спектр гамма-излучения, облученного суперконденсатора с наведенной гамма-активностью около $10^{7}$ Бк.

После реакторного облучения спустя 50 суток были выполнены измерения электрического напряжения суперконденсаторов в течение 70 суток (рис. 5). Результаты измерений свидетельствуют о нестабильности напряжения облу-

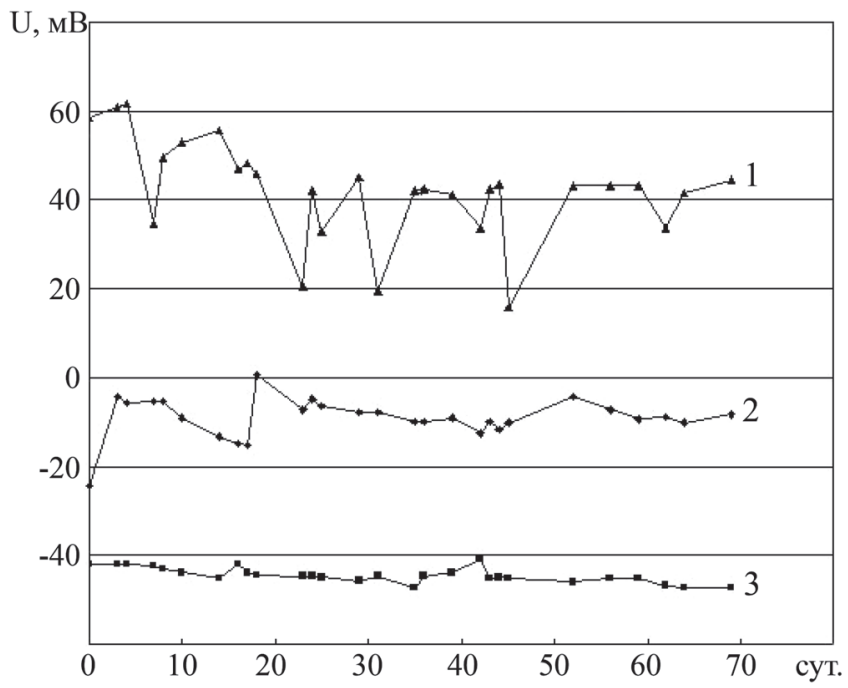

Рис. 5. Измерения разности потенциалов на обкладках суперконденсаторов спустя 50 суток после реакторного облучения с поглощенной дозой $2 \cdot 10^{12}$ н/см², 3 - необлученный образец ченных суперконденсаторов во времени. Колебания составляют 10-20 мВ. Для суперконденсатора, который не облучался, колебания разности потенциалов в течение времени наблюдения не превышают 5 мВ.

В суперконденсаторах двойной электрический слой с удельной площадью до $10^{3} \mathrm{M}^{2} / \Gamma$ образуется на поверхности наночастиц углеродного материала в результате адсорбции ионов из электролита или ориентировании полярных молекул на границе фаз. Один распад, например, ${ }^{14} \mathrm{C}$, приводит к перемещению через двойной электрический слой $10^{13}$ электронов в результате термоэлектронного переноса на границе электрода с электролитом. Если принять такое же число переноса электронов из электролита на электроды или с поверхности электродов в электролит в наблюдаемых облученных суперконденсаторах, то колебания до 20 мВ при емкости 22 Ф связаны с перераспределением заряда до 0,44 Кл $\left(\sim 3 \cdot 10^{18}\right.$ электронов) или с $\sim 3 \cdot 10^{5}$ событиями радиоактивного распада по одну из сторон относительно двойного электрического слоя. Полагая такие события пуассоновскими (дисперсия равна среднему), получим, что полное число событий за время между измерениями $\left(10^{5} \mathrm{c}\right)$ составляет $\sim 10^{11}$ или происходит с частотой около $10^{6} \mathrm{c}^{-1}$. С учетом наведенной внутренней $\beta$-активности $10^{7}$ Бк получаем разумную величину: 10 \% от всех делений изотопов происходят вблизи границ между электролитом и электродами, вблизи двойного электрического слоя. Такая оценка эксперименталь- 
ных данных вполне соответствует предложенному механизму локального термоэлектронного переноса в МДМ структурах и радиационно-индуцированному формированию двойного электрического слоя в суперконденсаторах.

\section{Выводы}

Исследования суперконденсаторов с активированными нейтронами введенных в электроды веществ (BN, $\mathrm{SrO})$ показали, что суперконденсаторы с внутренней радиоактивностью могут быть эффективными ИТРИ. Показано, что скорость зарядки ИТРИ увеличивается пропорционально активационной нейтронной дозе до $1,0 \cdot 10^{15} \mathrm{H} / \mathrm{cm}^{2}$. Скорость накопления заряда ИТРИ пропорциональна количеству $\beta$-активных изотопов ${ }^{14} \mathrm{C}$, образующихся в результате (n,p) реакции на азоте нанокристаллического BN, введенного в один из электродов.

Исследования скорости радиационно-индуцированного накопления заряда в ИТРИ и динамических электрических флуктуаций в суперконденсаторах после нейтронного облучения при учете выделяющейся при $\beta$-распаде ${ }^{14} \mathrm{C}$ и ${ }^{89} \mathrm{Sr}$ энергии исключают известные механизмы разделения заряда в конденсированной среде в результате ионизации и рождения вторичных электронов, образования электронов и дырок и т.п. Наиболее вероятный механизм разделения и накопления электрического заряда в ИТРИ связан с неравновесными термоэлектрическими явлениями в приэлектродных нанообластях, с термоэлектронным переносом на границе электрода с электролитом.

\section{Лuтература}

1. Кэбин Э. Радиоизотопные источники энергии и тепла. http://nuclphys.sinp.msu.ru/nuc_techn/ isotopes/index.html (обр. 15.01.2020)

2. Чернов В.А., Степанов В.А., Сигейкин Г.И., Прудников Н.В., Еремин В.П. Миниатюрные Российский химический журнал. 2016. T. LX. № 3. С. 20-25.

3. Степанов В.А., Чернов В.А., Паршиков Ю.Г, Лебедев В.П., Харанжевский Е.В. Изв. ВУЗов. Ядерная энергетика. 2018. № 1. С. 146-153.

4. Stepanov V.A., Chernov V.A., Parshikov Yu.G., Lebedev V.P., Kharanzhevskiy Y.V. Nuclear Energy and Technology. 2018. V. 4(3). P. 163-166. DOI: 10.3897/nucet.4.30780.

5. Степанов В.А., Лебедев В.П., Паршиков Ю.Г., Харанжевский E.B., Чернов В.А. Электродинамические эффекты в асимметричных суперконденсаторах после нейтронного облучения. / в кн.: «Технологии и материалы для экстремальных условий» (прогнозные исследования и инновационные разработки): материалы Всероссийской научной конференции под общей редакцией акад. Б.Ф.Мясоедова - М.: МЦАИ РАН, 2018. 376 с. - ISBN 978-5-4465-2049-7 / C. 100-105.

6. Чернов В.А., Степанов В.А., Прудников Н.В., Сигейкин Г.И., Леонова Е.A. Нано- и микросистемная техника. 2015. №2. С. 57-64.

7. Библиотека EXFOR. https://www-nds.iaea.org/exfor/ (обр. 01.13.2020).

8. Степанов В.А. ВАНТ Сер. Материаловедение и новые материалы. 2006. Вып. 1(66). С. 54-62.

9. Учайкин В.В., Амброзевич А.С., Сибатов Р.T. Амброзевич С.A., Морозова Е.В. Журнал технической физики. 2016. Т. 86. Вып. 2. С. 95-104.

10. Bertrand N., Sabatier J., Briat O., Vinassa J.M. Commun. Nonlinear Sci. Numer. Simul. 2010. V. 15. N. 5. P. $1327-1337$. 